Canadian University Music Review

Revue de musique des universités canadiennes

\title{
Un modèle du processus de composition musicale
}

\section{Martial Sauvé}

Numéro 5, 1984

URI : https://id.erudit.org/iderudit/1013941ar

DOI : https://doi.org/10.7202/1013941ar

Aller au sommaire du numéro

Éditeur(s)

Canadian University Music Society / Société de musique des universités

canadiennes

ISSN

0710-0353 (imprimé)

2291-2436 (numérique)

Découvrir la revue

Citer cet article

Sauvé, M. (1984). Un modèle du processus de composition musicale. Canadian University Music Review / Revue de musique des universités canadiennes, (5),

222-241. https://doi.org/10.7202/1013941ar

All Rights Reserved (C Canadian University Music Society / Société de musique des universités canadiennes, 1984
Ce document est protégé par la loi sur le droit d'auteur. L’utilisation des services d'Érudit (y compris la reproduction) est assujettie à sa politique d'utilisation que vous pouvez consulter en ligne.

https://apropos.erudit.org/fr/usagers/politique-dutilisation/ 


\title{
UN MODÈLE DU PROCESSUS DE COMPOSITION MUSICALE
}

\author{
Martial Sauvé
}

\section{Introduction}

$\mathrm{Au}$ moment où un compositeur de métier prend place devant un piano, une console de studio, ou encore un terminal d'ordinateur, afin de réaliser une œuvre commandée, s'enclenche un des plus nobles et des plus complexes mécanismes humains : le processus de composition musicale. Est-il possible de décrire et d'expliquer ce processus artistique? Quels en sont les éléments essentiels et comment fonctionnent-ils ensemble? Voilà les questions qui sont au centre de cette étude.

Bien qu'il existe de nombreux traités de composition (D'Indy, Fux, Hindemith, Křenek, etc.), il existe très peu d'études sur le processus de composition musicale. En 1947, Max Graf publiait From Beethoven to Shostakovich : The Psychology of the Composing Process. En 474 pages, il dépeint le processus de composition selon une méthode psychanalytique. Sans qu'elle ne soit restreinte à un individu, comme l'a fait récemment Marshall (1972) à l'égard de J.S. Bach, la thèse de Graf se fonde sur un certain "sentier " qui commence dans l'inconscient de l'être - où s'enracinent les instincts, le plus important étant, selon Graf, l'érotisme qui confère de "l'éclat au timbre de la musique " - au "royaume de la lumière " où "l'intellect arrange, organise et assemble les idées"(Graf $1947: 448)^{1}$.

Même si les études concernant le processus de composition musicale sont plutôt rares, il existe néanmoins de nombreux travaux à l'égard du processus de créativité en général (scientifique et artistique). Graham Wallas (1926) démontra que le processus de créativité se déroule en plusieurs étapes : 1) la PRÉPARATION : ici se formule progressivement un problème, une intention enclenchée par une motivation quelconque; 
2) l'INCUBATION : une période extrêmement fertile en idées pendant laquelle l'individu laisse voguer son imagination "en roue libre "; 3) l'ILLUMINATION : elle survient normalement après une période d'incubation et s'opère souvent sous le principe de la bisociation - chère à Arthur Kostler - qui consiste principalement à créer des analogies ; 4) la VÉRIFICATION : un peu à l'inverse de l'incubation, domine ici l'esprit critique de l'artiste qui évalue la situation et rejette les solutions banales, s'il y a lieu. Il résulte des recherches de Wallas que l'inspiration (troisième phase) n'est qu'une étape du processus de créativité. D'ailleurs, cet aspect du processus a toujours été au centre d'une publicité outrée dans l'histoire de la musique, publicité fallacieuse qui consiste à faire croire au grand public que l'inspiration est le nombril de l'art : «Elle n'est aucunement la condition préalable de l'acte créateur, mais une manifestation secondaire dans l'ordre du temps " (Stravinski $1945: 76$ ).

Nous n'aborderons pas ici le problème posé sous l'angle de la psychanalyse tel que proposé par Graf car cette perspective nous semble reposer sur une fable symbolique qui cloisonne l'activité créatrice en deux compartiments : l'instinct et l'intellect, distinction surannée qui existait entre les pensées sataniques du culte de Dionysos et les idées sobres du culte d'Apollon. À cet égard, Minsky souligne que :

Ces vieilles distinctions - l'affect, la raison, l'esthétique sont incontestablement une mauvause idée. On pourrait les comparer à la Terre, à l'Air et au Feu des alchimistes d'antan. Il nous faut des concepts bien meilleurs pour mettre en ouvre une chimie du psychisme (Minsky 1981:77).

Les phases essentielles du processus de composition sont vraisemblablement conformes aux grandes lignes tracées par Wallas, concernant la créativité. Toutefois, nous constatons dans cette étude que les étapes du processus de composition se succèdent en désordre et cela nous incite à proposer un modèle non-linéaire, mieux capable d'illustrer la complexité d'un comportement dynamique. Un modèle cognitif nous semble donc approprié. Bien entendu, un tel modèle n'est qu'une analogie circonspecte de la réalité mais, par ce paradigme, il devient possible de décrire les parties d'un système et d'expliquer, aussi peu soit-il, leur fonctionnement à l'intérieur d'un tout. Nous optons donc pour la philosophie de la "boîte blanche " dans laquelle interagissent des processus cognitifs tels la planification, l'idéation, l'organisation et l'écriture musicale ${ }^{2}$. 
Avant de présenter les détails de ce modèle, il importe d'en esquisser les frontières. Tout d'abord, il s'agit d'un modèle conjectural qui reste à être vérifié par des méthodes scientifiques en dépit du fait qu'il émane de recherches antérieures ${ }^{3}$, dans le domaine de la créativité et des travaux scientifiques de Flower et Hayes (1981) à propos du processus de composition littéraire. D’autre part, le modèle se confine à la performance de l'individu, c'est-à-dire qu'il illustre le comportement d'un compositeur ou d'une compositrice en action. D'autres aspects importants sont volontairement mis à l'écart comme l'apprentissage, la perception, la motivation et la personnalité car il nous apparaît nécessaire de restreindre le champ de cette étude.

\section{Le modèle : description des principales composantes}

Le modèle proposé, illustré brièvement à la figure ci-dessous, se décompose en trois régions. Tout d'abord, la partie supérieure de la figure - comprenant l'intention (objectifs, problème posé, etc.) et l'œuvre en cours - forme l'ENVIRONNEMENT DE LA TÂCHE. La région centrale (grand rectangle) comprend les PROCESSUS COMPOSITIONNELS de traduction (langage musical), révision et planification (but, esquisse, organisation et idéation), le tout orchestré par un CONTRÔLE ou MONITEUR. La MÉMOIRE LONG TERME, vaste réservoir de connaissances, complète ce schéma du modèle.

Dans cette section, nous décrirons chacune des composantes du système ainsi que l'articulation de celles-ci au sein d'un tout dynamique. 


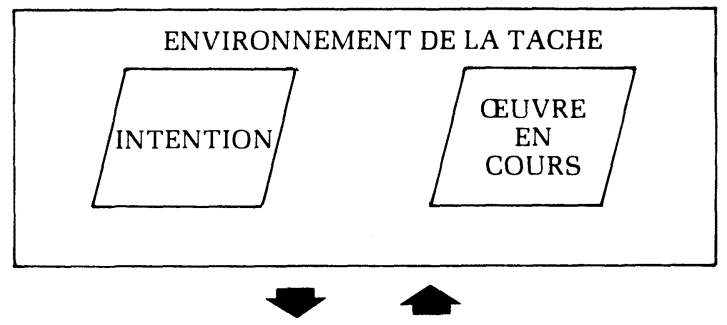

PROCESSUS COMPOSITIONNELS
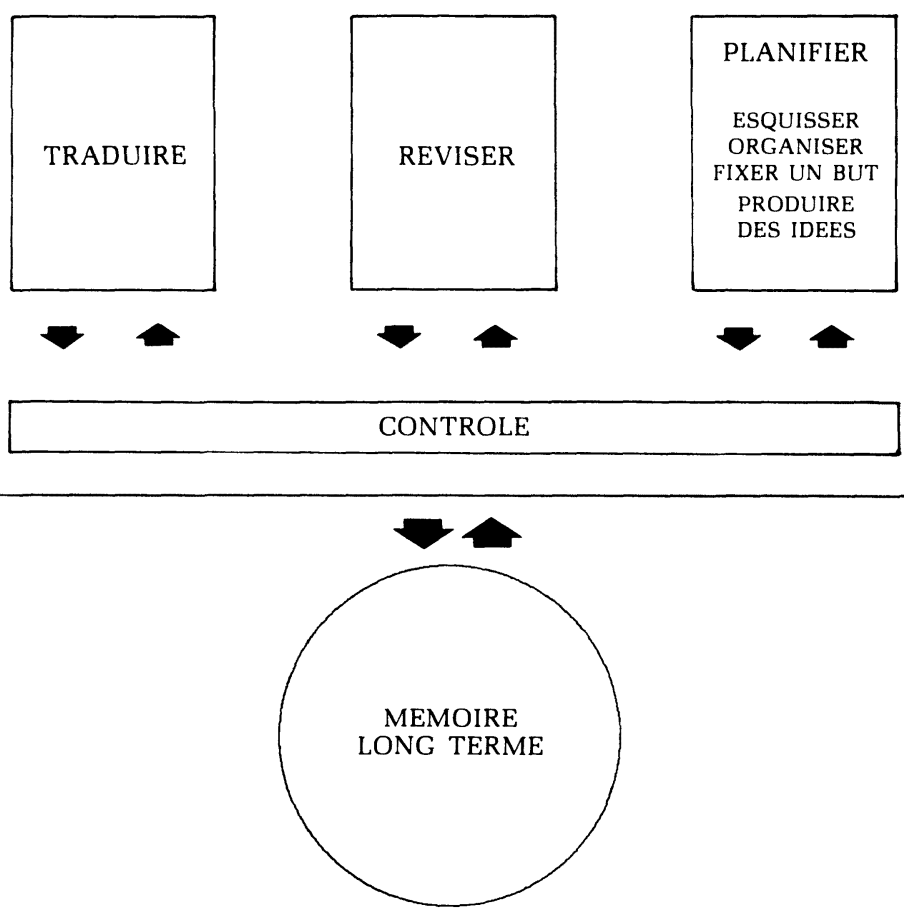

Modèle non-linéaire du processus de composition. Pendant ce processus, le compositeur traduit, planifie, revise, etc. constamment (ce va-et-vient est illustré par les flèches). 


\section{L'intention}

L'intention artistique, c'est une envie, une passion, un appétit, un but ou un problème formulé consciemment ou inconsciemment par l'artiste. Bien entendu, le compositeur ne parvient à formuler que vaguement l'intention car c'est souvent en travaillant d'autres aspects du mécanisme de composition que, soudain, elle se révèle avec plus de clarté. Écrire une sonate pour piano est un objectif; explorer les possibilités des sons multiphoniques à l'intérieur d'une œuvre pour quintette à vent, composer de la musique concrète avec un seul son, tenter d'éliminer sa personnalité de l'œuvre par le hasard (John Cage), exprimer des réminiscences affectives ou traduire une formule mathématique en musique, sont d'autres intentions possibles.

Pour formuler une intention, beaucoup d'artistes professionnels se tourneront vers les destinataires (auditeurs, interprètes, critiques, etc.) car s'attarder aux connaissances, besoins et attitudes de ceux-ci comporte certains avantages. L'altruisme favorise une certaine prise de conscience de l'ego : "C'est précisément parce qu'il considère l'attitude de l'auditeur que le compositeur devient conscient de son propre moi dans le processus de création " (Meyer 1956:41)4. Communiquer, voilà sans doute un autre avantage puisque la communication implique un terrain d'entente, une certaine relation entre l'artiste et son public afin de faciliter l'écoute et la compréhension de la musique.

\section{L'œuvre en cours}

Dans l'environnement de la tâche se trouve, en plus de l'intention du compositeur, la pièce en gestation ou l'œuvre en cours notée sur une partition (ou codée sur une bande dans le cas d'une musique électro-acoustique). Un tel échiquier influe sur le déroulement du processus puisqu'il s'impose comme contrainte - ce sentiment de l'étau qui se resserre, tout novice en contrepoint l'éprouve vivement dès qu'il expose le sujet d'une fugue. En se penchant sur son ouvre en cours, l'artiste est en mesure de réviser plusieurs aspects de celle-ci (organisation macroscopique et microscopique, concepts-clef, objectifs, etc.), mais il a la possibilité aussi d'utiliser l'œuvre en cours comme tremplin, comme source d'inspiration au prolongement de l'œuvre.

Il est convenu de distinguer deux types de mémoire: 
la mémoire court terme (MCT) et la mémoire long terme (MLT), vaste réservoir de connaissances acquises par le processus de l'apprentissage.

La connaissance " descriptive ${ }^{5}$ » est un type de connaissances de la mémoire long terme. Comme un banque de données, elle se rattache aux descriptions de concepts - motif, cadence, thème, série, progression, etc. Un musicien possède aussi un savoir qui lui permet d'exécuter des tâches, comme écrire une ouvre pour grand orchestre ou composer une sonate pour piano. Il s'agit là de la connaissance "procédurale ${ }^{6}$ " qui concerne le comment des choses. En plus des règles de syntaxe d'une langue musicale, la connaissance procédurale comprend des stratégies ou heuristiques asquises par l'expérience et employées pour oeuvrer non seulement au niveau de la syntaxe mais aussi pour planifier et réviser la pièce.

\section{Le processus de révision}

Le mot "réviser" vient du latin revisere qui veut dire "revenir voir». Le compositeur "revient voir» maintes fois pendant le processus et ce, pour évaluer ou continuer l'œuvre. Par exemple, Beethoven critiquait, vérifiait, évaluait, jugeait constamment son écriture musicale - le début du thème d'ouverture de l'air "Mir ist so wunderbar » de Fidelio a apparemment été l'objet de treize tentatives - comparativement à un J.S. Bach qui, toutefois, ne recopiait jamais une ouvre sans y apporter des changements, souvent mineurs. Et que dire du minutieux compositeur viennois Anton von Webern : des études de ses écrits révèlent que dans l'opus 24 , concerto pour neuf instruments, il aurait écrit au moins cinq versions des premières mesures.

Mais comment le musicien révise-t-il l'œuvre en cours? Par l'écoute. Pour les œuvres instrumentales et vocales relativement simples et bien tempérées, le piano demeure un outil valable de révision. Mais qu'en est-il des textures complexes telles les opéras ou les ouvres contemporaines pour grand orchestre? Comment écouter un fragment d'une partition en cours comprenant une section de trente vents qui évoluent en quarts de ton, accompagnée d'une section de quarante-huit cordes exécutant quarante-huit "glissandi " distincts, le tout chapeauté d'un chour de cent voix? Le recours à l'imagination est inéluctable. Ainsi, le compositeur imagine, autant que faire se peut, le résultat sonore de la partition en cours, la véritable 
écoute prenant place le soir de la première lorsqu'en principe l'œuvre est achevée - en principe, puisqu'il est toujours possible de réviser celle-ci après exécution : joué pour la première fois en 1845 à Dresde, l'opéra Tannhäuser de Richard Wagner fut remanié plusieurs fois par la suite, la dernière version étant celle présentée à Vienne en 1875. Il demeure que ce processus de révision diffère passablement de celui d'un J.S. Bach qui profitait d'un feed-back sonore pendant le processus de composition; il diffère aussi du processus de révision du poète qui voit défiler les mots au bout de sa plume ou celui de l'artiste peintre face à un tableau.

Il apparaît important de souligner, enfin, que le processus de révision a la particularité de pouvoir interrompre le processus de composition n'importe quand. Que ce soit pendant l'écriture d'un passage ou au moment de l'organisation d'une section, il est toujours possible pour le compositeur de s'arrêter et de prendre du recul, de "revenir voir».

\section{Le processus de traduction}

Pour le compositeur, traduire consiste à faire passer ses idées, émotions, images, etc. dans le langage musical, par l'intermédiaire d'un système de notation, s'il y a lieu.

Afin de traduire sa pensée, il utilise une langue musicale de son choix qui comporte habituellement un certain nombre de règles. Mais ce n'est là qu'un aspect de son activité. Comme le souligne Arthur Koestler (1968), il y a aussi dans toute activité un aspect variable : contraint par les règles du jeu, l'artiste a recours à des stratégies (connaissance procédurale) afin d'accomplir la tâche de traduction. Ce dernier aspect nous semble capital. À titre d'exemple, considérons le système tonal qui comporte plusieurs règles (contrepoint, harmonie, forme, etc.). Les grands courants historiques qui ont eu recours à ce système se sont caractérisés principalement par l'éventail de tactiques utilisées pour faire face aux différents problèmes musicaux - les règles tonales étant relativement les mêmes d'un courant à l'autre. Ainsi, sur le plan esthétique, par exemple, les Romantiques ont eu recours aux grands orchestres afin de créer des tensions dans le discours musical contrairement aux Impressionnistes qui composaient pour des ensembles réduits mais, par contre, profitaient d'une plus grande palette sonore afin d'accroître l'attention de l'auditeur. De même, à l'intérieur d'un courant musical, les stratèges se distinguent par leur 
éventail de tactiques. Par exemple, un des objectifs visés par le courant de musique aléatoire, créé vers 1945, consiste à utiliser le hasard afin de produire les hauteurs, durées et intensités d'une pièce. Mais si la règle est d'utiliser le hasard, alors comment l'engendrer? La tactique d'un John Cage se fondera sur l'emploi de l'ouvrage chinois I-Ching tandis que celle d'un lannis Xenakis se fondera sur l'utilisation de lois mathématiques du domaine des probabilités. C'est donc sous la pression de contraintes inhérentes à une langue musicale et par le maniement de stratégies adéquates que le compositeur traduit sa pensée.

De tous les mécanismes du processus de composition, le processus de traduction est probablement celui qui engendre le plus de frustration chez le compositeur novice qui ne maîtrise pas encore tous les rudiments de la langue musicale choisie ni un catalogue de stratégies efficaces pour affronter les divers problèmes d'écriture. Le novice est plutôt engagé dans un processus d'apprentissage qui, à vrai dire, freine sa performance artistique. Tout comme un joueur d'échec novice, il peut difficilement "gagner " puisqu'il doit nécessairement consacrer beaucoup de temps à apprendre les règles fondamentales. Traduire est largement une affaire de métier qui demande une solide technique et cela ne s'acquiert qu'avec les années.

Un aspect important du processus de traduction et qui caractérise la musique savante occidentale depuis l'antiquité grecque, consiste à utiliser un système de notation approprié afin de permettre à un interprète ou à un ensemble d'exécuter l'œuvre. La notation utilisée dépendra des contraintes imposées par la langue musicale choisie ainsi que des objectifs formulés par l'individu. Étant le fruit de plusieurs siècles de pratique musicale, le système traditionnel - portée, clefs, figures de notes, barres de mesure, etc. - s'avère un système adéquat pour bon nombre de musiques du $\mathrm{XX}^{\mathrm{c}}$ siècle issues de diverses tendances (expressionnisme, néo-classicisme, atonalisme et sérialisme). D'autres courants, tels la musique aléatoire, ont recours à une notation différente, parfois très imagée : dès 1951, le compositeur américain Morton Feldman écrivait Intersection $I$, pour orchestre, en précisant les registres haut, moyen et grave sans toutefois spécifier les hauteurs des notes - ce choix étant réservé aux interprètes. Par ailleurs, l'avènement de la musique électro-acoustique a suscité un profond changement de notation par rapport au système traditionnel. Cela est dû, pour une large part, à la quasi impossibilité de noter 
en symboles conventionnels des timbres complexes comme le "bruit blanc » ou des valeurs d'intensité comme le "décibel ». Il faut souligner toutefois que cette notation électro-acoustique varie d'un compositeur à l'autre et il ne semble pas y avoir encore de consensus autour d'un système commun partagé par un grand nombre d'individus - ce qui, en général, ne pose pas de problèmes à l'artiste qui monte la totalité de l'ouvre en studio mais dans l'éventualité où cette musique demande à être exécutée en concert, par des interprètes "jouant " divers dispositifs électroniques, cela peut devenir une situation problématique sur le plan de l'efficacité d'exécution (de même pour une partition de musique "mixte " avec repères de synchronisation pour l'interprète et la bande).

\section{Le processus de planification}

Pendant le processus de planification, le compositeur construit une représentation interne des idées, émotions, images, etc. qu'il aura éventuellement à faire passer dans le langage musical, lors du processus de traduction. Ce processus comprend plusieurs sous-processus : fixer un but, esquisser, produire des idées et organiser.

\section{A. Fixer un but}

Les intentions formulées consciemment ou inconsciemment par les compositeurs dans l'environnement de la tâche, se résument souvent à des énoncés abstraits et imprécis du genre "écrire une pièce pour clavier " ou "composer une oeuvre qui évoque un souvenir ». Pendant le sous-processus de planification fixer un but, le compositeur se définit un objectif concret, par exemple " écrire une pièce pour piano d'une durée de cinq minutes selon une forme ABA - une introduction plutôt paisible suivie de la partie $B$ - très agitée - puis une réitération plus ou moins précise de l'introduction, etc. ". Il est possible aussi que, de ce but opérationnel, découle plusieurs sous-objectifs : " une introduction de seize mesures construite à l'aide d'une série accompagnée de sa version rétrograde ... » forme un autre objectif qui découle directement d'un objectif de plus haut niveau. En plus de fournir un cadre à la pensée, lors du processus d'idéation, l'objectif opérationnel est à un compositeur ce qu'un ancrage est à l'alpiniste. Il s'agit donc d'un outil de travail concret grâce auquel l'artiste peut avancer.

Quoiqu'il existe des objectifs préfabriqués en permanence 
dans la mémoire long terme du compositeur, plusieurs se formulent provisoirement ou définitivement pendant le processus de composition. Après avoir écrit une introduction, le compositeur peut décider de la modifier ou décider de substituer à la forme ABA une forme plus complexe. Les objectifs concrets sont donc susceptibles d'être formulés, reformulés ou rejetés et ce, avant, pendant ou après le processus de traduction.

\section{B. Esquisser}

Tout comme les architectes, les compositeurs tirent parti de croquis variés pour édifier leurs œuvres. Ces esquisses prennent tantôt la forme de dessins et de notes écrites, tantôt de graphiques et de fragments musicaux. De tous les principaux compositeurs de musique tonale, il appert que Beethoven fut celui qui en fit un usage ample et diversifié. Il existe, selon Joseph Schmidt-Gorg (voir Forte 1974:3), 5000 pages de croquis esquissés par l'auteur - ce qui est volumineux comparativement à ses contemporains et successeurs -, ceux-ci s'échelonnant du simple motif au premier jet de mouvements entiers. Étant donné qu'elle se monte, se modifie ou se rejette rapidement, l'esquisse permet à l'artiste de fixer, organiser, produire ainsi que réviser efficacement ses idées et, comme une photographie du contenu mental, elle devient un stimulus visuel pratique qui remplace l'effort préalablement déployé pour appréhender ce contenu. Il faut souligner, par ailleurs, que l'emploi du croquis n'est pas une nécessité car cela dépend de la nature du projet artistique et de la personnalité du compositeur - plutôt improvisateur, Chostakovitch utilisait rarement les esquisses pour écrire ses ouvres. Mais lorsque les compositeurs s'engagent dans des œuvres de grande envergure, comme les opéras ou les ouvres pour chour et grand orchestre, les croquis leur sont parfois d'un grand secours.

\section{Produire des idées}

À la lumière d'un but opérationnel, il est souvent nécessaire de produire des idées pour atteindre ce but, soit en restituant des concepts qui sont déjà logés dans la mémoire long terme, soit en créant de nouveaux concepts par l'imagination. Choisir une forme ABA en tant que structure macroscopique de l'œuvre ou façonner l'aspect dramatique de celle-ci selon des expériences émotives ressenties au cours de l'existence - par exemple, 
la hantise de la mort chez Gustave Mahler - sont des idées qui peuvent découler de ce processus. Bref, les idées sont les blocs de l'architecture musicale et, en principe, elles se rattachent à tous les aspects de l'édifice sonore (syntaxe, sémantique, esthétique, etc.)

Bien que plusieurs compositeurs les utilisent inconsciemment, il existe un grand nombre de techniques d'idéation aujourd'hui (voir Bouchard 1971), grâce à l'apport considérable de recherches américaines dans le domaine de la créativité depuis la fin de la dernière guerre. La méthode brainstorming est vraisemblablement la plus connue pour produire des idées nouvelles. Alex Osborn, le père de cette technique, l'a conçue comme une stratégie pour attaquer et éventuellement résoudre un problème; elle consiste principalement à laisser l'imagination en "roue libre », face à un problème, en formulant toutes les idées qui nous viennent à l'esprit et en s'abstenant de les critiquer - cette technique fonctionne aussi bien chez un individu qu'un groupe d'individus. Il s'agit donc d'une méthode efficace de production d'idées qui diminue les inhibitions provoquées par l'auto-critique. Au fond, plusieurs ouvres de John Cage, composées aléatoirement, sont ni plus ni moins le résultat de vastes brainstorming comme en fait foi sa pièce électro-acoustique HPSCHD pour sept clavecins et cinquante et une bandes magnétiques ${ }^{7}$. À l'exception de Cage, qui a subi l'influence du Zen, la plupart des compositeurs occidentaux toutefois utilisent le brainstorming comme une stratégie d'idéation préalable au processus d'organisation.

\section{Organiser}

Puisque l'ensemble des idées issues du processus d'idéation est rarement organisé logiquement alors le rôle du processus de l'organisation est d'assembler ces idées dans un tout organique cohérent.

Il est convenu de distinguer deux niveaux d'organisation dans une ouvre. Le premier niveau se rapporte à l'ordre de présentation des événements ${ }^{8}$ musicaux alors que le second consiste à distinguer les événements principaux des événements secondaires, à déterminer comment ils sont liés les uns aux autres et à former de nouveaux concepts en groupant plusieurs événements. Les compositeurs utilisent fréquemment les structures hiérarchisées, qu'on appelle arbres, comme stratégie d'organisation car ces hiérarchies isolent les événements principaux des événements subordonnés et elles facilitent la création 
de nouveaux concepts. Une phrase musicale composée d'un antécédent et d'un conséquent est un exemple de structure hiérarchisée : l'événement principal (phrase) correspond à la concaténation de deux sous-événements, l'antécédent et le conséquent. De plus, de ces sous-concepts peuvent découler des événements subordonnés comme le motif formant ainsi une hiérarchie à plusieurs niveaux. Bien que la musique tonale soit fortement hiérarchisée dans son organisation, le principe d'arborescence ne se limite pas uniquement à cette musique, comme en fait foi son anthithèse, la musique atonale : la forme macroscopique de Structure la, pour deux pianos de Pierre Boulez, comprend deux parties et chacune d'elles se subdivise en plusieurs sections formant ainsi une organisation en forme d'arbre. D'autre part, dans le domaine de la musique électroacoustique, la construction de Visage de Luciano Berio est aussi un bel exemple de structure hiérarchisée - Berio s'est d'ailleurs inspiré de la structure du langage littéraire pour construire cette pièce (il a inventé des syllabes qui découlent d'un agencement de phonèmes initiaux et avec celles-ci, il a créé des mots).

Par ailleurs, la manière dont ces hiérarchies sont érigées varie selon le style des compositeurs. Certains préféreront procéder du haut vers le bas de l'arbre (top-down), en d'autres mots, il imaginent avant tout la forme globale d'un événement pour ensuite le subdiviser en sous-événements et ainsi de suite jusqu'au plus bas niveau de l'arbre qui correspond à la musique proprement dite. Inversement, il est possible de construire ces arbres du bas vers le haut (bottom-up) en commençant, par exemple, avec un simple motif qui, par transformation, engendrera d'autres événements, de même niveau, ceux-ci pouvant être regroupés pour former une catégorie à un niveau supérieur de la hiérarchie. C'est dans cette dernière perspective, par exemple, qu'Edgar Varèse édifiait ses ouvres, « (...) concevant la forme musicale comme une résultante, le résultat d'un processus (...) " (voir Ouellette $1966: 70$ ). Il apparaît toutefois que les compositeurs adoptent le plus souvent une méthode souple d'organisation qui consiste à combiner les démarches ascendante et descendante plutôt que de se limiter à l'une d'entre elles.

Mais pourquoi utilise-t-on des structures arborescentes en tant qu'outil d'organisation et ce, depuis plusieurs siècles de musique occidentale? Il semble qu'un élément de réponse se trouve du côté de l'auditeur. Qu'il s'agisse de traiter de n'importe quel type d'information (littéraire, auditive, visuelle, etc.), 
l'humain digère celle-ci par "morceaux " à cause des limites qu'impose la mémoire court terme (MCT) - sorte d'espace de travail (zone tampon) dont la capacité d'absorption d'éléments simultanés est limitée ${ }^{9}$ et dans laquelle prend place le traitement de l'information musicale (percevoir, comprendre et retenir la musique). Par exemple, au lieu de dire "j'entends un accord du cinquième degré à l'état fondamental, comprenant la sensible au soprano et la septième à l'alto, suivi de l'accord du premier degré, comprenant la tonique au soprano et la tierce à l'alto ", nous dirons simplement « cadence parfaite». En groupant ainsi l'information autour de concepts-clef (morceaux), organisés hiérarchiquement, l'auditeur distingue les points essentiels des détails secondaires et réussit ingénieusement à comprendre et retenir la musique tout en surmontant le problème de la mémoire court terme. De là, il est sans doute possible qu'une musique organisée hiérarchiquement soit davantage comprise et retenue puisqu'elle se présente à l'auditeur par fragment, ce qui pourrait impliquer, par surcroît, une meilleure communication entre le compositeur et les destinataires.

Par ailleurs, il n'y a pas de moment précis pour organiser les idées, lors du processus de composition. Cela dépend des objectifs visés ainsi que du type de langue musicale employée. D'aucuns préfèrent organiser tous les aspects de l'œuvre jusqu'aux détails infinitésimaux avant d'écrire une note. Dans l'histoire de la musique sérielle européenne se trouvent plusieurs exemples d'organisation totale où les paramètres de durée, hauteurs, "tempi », registres d'octave, intensités, timbres, formes, etc. étaient prédéterminés - selon les principaux stratèges sérialistes, ce totalitarisme se justifiait par leur désir de créer une nouvelle musique détachée du passé et réalisée par une pensée objective. D’autres organisent les événements musicaux tout au long du processus compositionnel, préférant une organisation formelle souple qui leur sert de cadre à la pensée et qui leur permet une plus grand part de subjectivité. La musique «stochastique " de Xenakis (1963) découle en principe de cette intention. Dans une ouvre comme Achorripsis, pour orchestre à cordes, il n'y a pas, à vrai dire, d'organisation syntaxique prédéterminée. Toutefois, la forme macroscopique et microscopique de l'œuvre est soumise à une organisation « hors-temps » modelée par des lois mathématiques du domaine des probabilités. 


\section{Le contrôle}

Les processus de traduction, révision et planification sont orchestrés sous la baguette du compositeur qui contrôle le temps alloué à un mécanisme avant de passer au suivant. Cette structure de contrôle varie selon les objectifs et le style de l'individu. Par exemple, certains compositeurs consacrent beaucoup de temps à planifier avant de traduire tandis que d'autres aiment mieux écrire rapidement, quitte à consacrer plus de temps au processus de révision. En fin de compte, la qualité et l'efficacité de la performance compositionnelle de l'individu dépend certainement de son habileté à orchester ces différents processus.

\section{Le modèle : fonctionnement}

Après avoir décrit les parties essentielles du modèle du processus de composition, il apparaît important ici de présenter une ébauche de leur fonctionnement à l'intérieur d'un tout dynamique. Les recherches de Flower et Hayes (1981) ont démontré que le processus de composition littéraire s'explique par un modèle non linéaire. Intuitivement, cela nous apparaît vrai à l'égard de la composition musicale. En effet, les compositeurs réalisent rarement les étapes de planification, de traduction et de révision successivement, d'un seul jet, sans détour. Les compositeurs évoluent comme les alpinistes ; ils rencontrent une multitude d'obstacles qui les contraignent à se déplacer dans tous les sens; ils planifient, traduisent et révisent constamment. Pour illustrer ce comportement, nous reproduisons ici un protocole verbal rapporté par Reitman : il a demandé à un compositeur de métier d'écrire une fugue et de verbaliser sa pensée pendant qu'il compose. L'extrait, ci-dessous, est un compte rendu, mot à mot, de l'activité créatrice du compositeur au moment où l'exposition du sujet, à la deuxième voix, est complétée, son intention courante étant de trouver une suite convenable pour la première voix (nous indiquons, entre crochets, les processus impliqués) :

Ceci est une question d'inspiration. J'ai besoin maintenant d'une idée qui est presqu'aussi bonne - combinaisons intéressantes d'intervalles et structure rythmique - que le sujet l'était et qui deviendra un contre-sujet ou simplement du bon contrepoint, parce qu'on ne se préoccupe plus du sujet (joue), il est répété. Et nous devons trouver quelque chose ... [but] on n'a qu'à jouer (rejoue les dernières notes du sujet). Il y a un problème pianistique (joue) [révision]. Nous devons 
trouver une bonne cohérence de tension dans les relations d'intervalles entre le sujet et la nouvelle contre-mélodie [but] (joue) ... oui [idéation] ..., oh, non [révision] (joue) [idéation] oui, ça va probablement marcher (joue) [traduction]. Voyons ce qui arrive avec ça (joue). Maintenant, nous avons un nouveau motif, da diii (chante et joue) [révision]. Qu'est-ce qu'on devrait faire avec le nouveau motif ? Il 'n'est pas encore très utile [but] (joue). Uh, quelque chose comme une syncope [idéation], da-daa, dii (chante et joue) [traduction] [révision]. Le rythme doit être différent du sujet tout en étant une idée distincte, une idée rythmique [but] (joue, compte). Poursuivant l'idée de syncope ... (joue) [idéation]. Ça ne marchera pas, parce que c'est une répétition du sujet lui-même (efface) [révision]. Je pense qu'on abandonne l'idée d'une syncope pour éviter la répétition du sujet ... et essayer de faire quelque chose de plus pianistique ... l'idée c'est d'avoir un rythme plus complexe contre le sujet qui est plutôt strict ... définitivement binaire [but] (joue, compte). Possibilité de quelque chose en triolets dans la contre-mélodie ... [idéation] [traduction], mais ça pourrait devenir trop compliqué sitôt dans la pièce (efface) [révision]. On pourrait essayer une syncope plus rapide, avec un effet pianistique ... [idéation] et on s'achemine vers un style comprenant de grands intervalles, des intervalles de plus en plus grands dans la contre-mélodie (joue) [révision]. Et ceci pourrait devenir un motif ... on dirait que oui [idéation] ... Maintenant, nous avons un motif potentiel (joue, compte) [traduction] [révision]. De plus, il m’apparaît correct et un peu plus pianistique que des simples notes (joue) [révision]. Maintenant, voyons l'ensemble (joue la première et deuxième voix simultanément) oui, ceci ira bien avec le sujet [révision] (Reitman $1965: 167-168)^{10}$.

Ce protocole illustre bien, nous semble-t-il, le dynamisme du modèle proposé et l'articulation possible des différents processus orchestrés par un compositeur. Pour aller plus loin, nous dirons que les processus compositionnels s'apparentent aux procédures d'un programme informatique : à sa convenance, le compositeur " appelle » les procédures par le biais du contrôle ou «moniteur»; il peut aussi, de l'intérieur d'une procédure, en "appeler » une autre, y compris la même (récursivité), ou "appeler " une version réduite de tout le modèle du processus, s'il y a lieu. Ces processus peuvent donc être emboîtés les uns dans les autres. Dans le protocole verbal ci-dessus, il est constaté, après analyse, que le compositeur scinde l'intention initiale (écriture du motif contre-sujet) en deux sous-tâches opération- 
nelles : pour une part, trouver les hauteurs des notes du motif et, pour l'autre, trouver la structure rythmique de celui-ci. Puis, le compositeur applique une version réduite du modèle dans les deux cas; il planifie, révise et traduit constamment.

\section{Conclusion}

Nous avons proposé, dans cette étude, un modèle dynamique du processus de composition selon une approche liée au domaine de la psychologie cognitive, approche qui tend à l'explication des mécanismes de création plutôt qu'à leur mystification. En somme, le système proposé se compose essentiellement de processus compositionnels (planification, révision et traduction) orchestrés au gré du compositeur. De plus, il ressort de cette étude que le processus de composition se déroule de façon désordonnée et imprévisible comme à l'intérieur d'un labyrinthe ; fixer un but, organiser les idées, réviser les concepts, traduire en notation musicale, formuler une intention, esquisser un plan, produire des idées, sont des actions que réitère constamment le compositeur pendant ce processus et qu'il applique à tous les niveaux de la composition, microscopique ou macroscopique.

Malgré le rôle incontestable qu'occupent l'apprentissage, la motivation, la perception et la personnalité du compositeur lors du processus de composition, il nous apparaissait primordial d'étudier avant tout la performance de l'individu. Par ailleurs, ce modèle de performance découle, en partie, de recherches scientifiques menées dans le domaine du processus de composition littéraire. Même si notre analyse d'un protocole verbal semble confirmer l'authenticité du modèle, il demeure qu'une investigation plus poussée, selon des méthodes scientifiques, serait souhaitable afin d'en confirmer ou non l'exactitude.

La possibilité de simuler le processus de composition, en tout ou en partie, semble une conséquence importante de cette étude. Un programme informatique de composition conçu à l'image de ce modèle pourra prendre en charge certains aspects fastidieux de la composition, tels l'organisation et la traduction, accélérant ainsi avantageusement, le processus de composition ${ }^{11}$. Si l'on songe aux récent systèmes-experts qui sont parmi les succès les plus significatifs du domaine de l'intelligence artificielle - tel l'étonnant programme AM de Douglas Lenat (1976) qui synthétise des découvertes mathématiques - alors la simulation totale du processus de composition ou l'édification d'un 
" compositeur artificiel » ne relève peut-être pas de l'utopie. Toutefois, ce n'est que lorsque nous disposerons d'une théorie robuste et pénétrante qui coiffe tous les aspects du processus de composition (planification, révision, traduction, motivation, apprentissage, personnalité, etc.) que vraisemblablement se réalisera ce genre de projet. Enfin, une autre implication de ce modèle se situe dans le domaine de l'enseignement de la composition musicale : chercher à identifier et à comprendre la substance de l'expertise des compositeurs et tenter de décrire ainsi que d'expliquer les mécanismes fondamentaux qu'on retrouve derrière leur processus créatif, ne peut qu'être bénéfique pour les novices. 


\section{NOTES}

1. "This path leads from the dark regions of unconscious soul life, where the roots of instincts reach deep into the psyche of man, to the realm of light where intellect arranges, organizes and joins the ideas." "

2. Le recours à des principes de la psychologie cognitive pour tenter de décrire certains aspects de l'activité musicale n'est pas nouveau; parmi ceux qui ouvrent dans cette perspective, citons Laske (1977) qui propose une approche cognitive à la musicologie.

3. Cf. Sauvé (1980).

4. "It is precisely because he is continually taking the attitude of the listener that the composer becomes aware and conscious of his own self, his ego, in the process of creation."

5. "Descriptive knowledge ".

6. "Procedural knowledge».

7. Cette pièce a été réalisée avec Lejaren Hiller.

8. Nous utilisons le terme événement pour signifier toute entité musicale telle un silence, un objet sonore, une note ou un groupe de notes (motif, thème, mouvement, pièce, etc.).

9. Miller (1956) estime que la limite est de sept plus ou moins deux unités d'information.

10. "This has to be a matter of inspiration. I need now an idea, that's almost as good in profile and the interesting combinations of intervals and rhythmic structure as the original [theme] was, which will either become a countersubject or become simply good counterpoint, because we no longer care about the theme (plays), it's being repeated. And we have to work something.... this just takes some playing actually (replays concluding notes of subject). There's a pianistic problem (plays). Hmm, we have to get the proper consistency of tension in the interval relationships between the theme and the new counter melody, (plays) ... yes ... oh, no (plays). Yes, that will probably work (plays). Let's see what happens with that (plays). Now we have a new motive, da diii (sings and plays). What shall we do with the new motive. It doesn't do anything yet (plays). Uh, some kind of syncopation, da- daa, dii (sings and plays). The rhythm has to be different from the theme, and yet hold itself as an idea, as a rhythmic idea (plays, counting). Carrying out the idea of enlarged syncopation ... (plays). That won't work, because it's repetitive of the theme itself. (...) (Erasing) I think we'll abandon the idea of the enlarged syncopation to get away from repetition of theme ... and try to do something more pianistic... The idea comes of getting a more complex rhythmic situation against the theme, which is rather rigid ... a very definite duple feeling (plays, counting). Possibility of something in the counter melody in triplets . . . but it may become too complicated to work with 
so soon in the piece (erasing). We can try a quicker syncopation, with pianistic effect ... and we get into the stylistic process of large intervals, larger and larger intervals in the counter melody . . . (plays). And that may become a motive... looks like it does ... sketch it out to .... Now we have a motive that may be something (plays, counting). A certain interest in that too, and which is also a bit more pianistic than single notes (plays). Now here, let's see how this goes together (plays first and second voices together). Yes, that will go well with this same thing that we had at the theme."

11. À l'égard de la mécanisation de certains aspects du processus, voir Sauvé (1979).

\section{RÉFÉRENCES}

BOUCHARD, M.

1971: Processus de l'innovation. Montréal : École d'Architecture, Faculté de l'Aménagement, Université de Montréal (inédit).

FLOWER, L. et HAYES, J.R.

1981: "A Cognitive Process Theory of Writing ", College

FORTE, A. Composition and Communication, XXXII/4, 365-387.

1974: The Compositional Matrix. New York : Da Capo Press. GRAF, M.

1947: From Beethoven to Shostakovitch: The Psychology of the Composing Process. New York : Philosophical Library.

KCESTLER, A.

1968: The Ghost in the Machine. New York: MacMillan. LASKE, O.

1977 : Music, Memory, and Thought : Explorations in Cognitive Musicology. Ann Arbor: University Microfilms International.

LENAT, D.B.

1976: AM : An Artificial Intelligence Approach to Discovery in Mathematics as Heuristic Search. SAIL AIM-286, Artificial Intelligence Laboratory, Stanford University.

MARSHALL, R.L.

1972 : The Compositional Process of J.S. Bach. 2 vols. Princeton : Princeton University Press.

MEYER, L.B.

1956 : Emotion and Meaning in Music. Chicago : University of Chicago Press. 
MILLER, G.A.

1956: "The Magical Number Seven, Plus or Minus Two: Some Limits on Our Capacity for Processing Information ", Psychological Review, LXIII/2, 81-97.

MINSKY, M.

1981: "La musique, les structures mentales et le sens », in M. Battier et al., Le compositeur et l'ordinateur. Paris : IRCAM, 56-81.

OUELLETTE, F.

1966 : Edgar Varèse. Paris-Montréal : Seghers - H.M.H.

REITMAN, W.R.

1965: Cognition and Thought: An Information Processing Approach. New York : John Wiley.

SAUVÉ, M.

1979: "Époques : musique électro-acoustique numérique ". Mémoire de maîtrise inédit (avec bande et partition). Montréal : Université de Montréal.

1980 : Vers un système de créativité artificielle. Document inédit.

STRAVINSKI, I.

1945 : Poétique musicale, $5^{\mathrm{e}}$ éd. Paris : Janin.

WALLAS, G.

1926: The Art of Thought. New York : Harcourt-Brace.

XENAKIS, I.

1963 : Musiques formelles. Paris : Richard-Masse. 